\title{
A two-centre study of risk factors involved in renal deterioration in neurogenic bladder in children after myelomeningocele
}

\author{
Joanna Bagińska', Monika Miklaszewska², Alicja Liszewska', Magdalena Deluga', \\ Agata Korzeniecka-Kozerska' \\ 'Department of Paediatric Nephrology, Medical University of Bialystok, Bialystok, Poland \\ ${ }^{2}$ Department of Paediatric Nephrology, Jagiellonian University Medical College, Cracow, Poland
}

\section{ABSTRACT}

Introduction: Myelomeningocele (MMC) is the first cause of neurogenic bladder in children. Adequate nephrourological management is crucial to prevent renal damage and bladder dysfunction. In the last decade, the prognosis of MMC children has dramatically improved, but can we do better? We wanted to detect risk factors involved in renal deterioration in MMC patients.

Material and methods: This retrospective analysis was based on 178 children with MMC (99 girls and 79 boys) from two Polish hospitals, in Bialystok and Cracow. The children's medical records were analysed to determine sex, age, and kidney and urinary tract ultrasound, voiding cystourethrogram, and urodynamic findings. The number of clean intermittent catheterisations performed per day, cystatin C, serum creatinine, urea, and glomerular filtration rate (GFR) Schwartz were also recorded. The Hoffer scale was used to assess MMC children's motor function.

Results: Of the 111 from Bialystok and 67 children from Cracow, with a median age of nine years, 45 (25\%) had vesicoureteral reflux. The most common urodynamic finding was overactive bladder with a prevalence of $59 \%$. There were statistically significant differences between girls and boys, including the presence of reflux, increased echogenicity of the kidneys' parenchyma in ultrasound examination and cystometric capacity in urodynamic study. There were statistically significant differences between the Hoffer scale groups, including GFR Schwartz. The number of performed urodynamics correlated with GFR Schwartz, serum concentrations of cystatin $\mathrm{C}$, and creatinine.

Conclusions: Potential risk factors of renal deterioration in children with MMC are abnormalities in ultrasound, the presence of high-grade vesicoureteral reflux in cystogram, increased bladder pressure on urodynamics, hyperfiltration, and increased levels of cystatin C. Urodynamic evaluation may play a role in the management of children with MMC by identifying those with increased bladder pressures. Frequent urodynamic assessment may contribute to prevention of renal function in children with MMC.

\section{KEY WORDS:}

myelomeningocele, neurogenic bladder, urodynamics, vesicoureteral reflux.

\section{ADDRESS FOR CORRESPONDENCE:}

Joanna Bagińska, Department of Paediatric Nephrology, Medical University of Bialystok, 17 Waszyngtona St., 15-274 Bialystok, ORCID: 0000-0002-6912-9502, e-mail: joasiabaginska14@wp.pl 


\section{INTRODUCTION}

Myelomeningocele (MMC) remains the most severe form of neural tube defects, with an estimated prevalence of one in 700 live births, involving dysfunctions of many organs and structures. Nervous, skeletal, and urinary are the most affected systems. Lifelong follow-up in many specialist clinics should be focused on, maintaining the correct function of these organs and systems $[1,2]$.

Our study was performed in paediatric nephrology departments in two Polish hospitals. We concentrated on nephrological problems in children with MMC, including neurogenic bladder. This results from interference with the normal nerve pathways associated with urination. MMC in children is the most common cause of neurogenic bladder, which is an important risk factor of secondary upper urinary tract damage. Hence, neurogenic bladder patients require close monitoring [3-5]. It is crucial to find a proper means of early identification of children with high risk of kidney function deterioration. Adequate follow-up of MMC includes renal and bladder ultrasound, measurement of residual urine, determination of serum creatinine levels, voiding cystogram, urodynamic study, and subsequent treatment.

There are only a few studies, mostly published before 2000, that focus on risk factors involved in renal deterioration in children with MMC. Due to a lack of recent literature, we wanted to fill that gap. In our study, we evaluated the kidney and urinary tract and voiding cystourethrogram as well as urodynamic findings, to detect early symptoms of renal deterioration in children with MMC. Adequate nephrourological management is important to improve the quality of MMC children's lives and to prevent both renal damage and bladder dysfunction $[6,7]$. Thanks to a multidisciplinary approach in the last decade, the prognosis of MMC in children has substantially improved, but can we further enhance the quality of their lives? We aimed to detect risk factors involved in renal deterioration in MMC patients by evaluating the urinary tract through ultrasound, voiding cystourethrogram, and urodynamic findings in children with MMC.

\section{MATERIAL AND METHODS}

This retrospective analysis was based on 178 children with MMC from two Polish hospitals: 111 from the Department of Paediatrics and Nephrology, Medical University of Bialystok, and 67 children from the Paediatric Nephrology Department in Cracow, Jagiellonian University Medical College. The study was performed over a period of 10 years, between 2007 and 2017. The inclusion criteria for the study group were as follows: 1) patients aged 1-18 years with neurogenic bladder after MMC, and 2) children with complete nephrological assessment: urinary tract ultrasound, voiding cystourethrogram, and urodynamics performed during the study period. The ex- clusion criterion was: patients with neurogenic bladder of different aetiology than MMC.

The children's medical records were analysed to determine sex, age, anthropometric parameters, number of clean intermittent catheterisations (CIC) performed per day, serum cystatin $C$, creatinine, and urea. The glomerular filtration rate (GFR) was calculated with the new Schwartz equation using serum creatinine and the patient's height. Normal range for GFR Schwartz was defined as $90-140 \mathrm{ml} / \mathrm{min}$ per $1.73 \mathrm{~m}^{2}$. Renal deterioration was defined as GFR $<90 \mathrm{ml} / \mathrm{min}$ per $1.73 \mathrm{~m}^{2}$ and GFR $\geq 140 \mathrm{ml} / \mathrm{min}$ per $1.73 \mathrm{~m}^{2}$ (glomerular hyperfiltration) [8].

The ambulatory function of MMC patients was defined according to the Hoffer scale (HS) using the four categories of community: $1 \mathrm{HS}$ - nonambulatory, $2 \mathrm{HS}$ - nonfunctional ambulatory, 3HS - household walkers, 4HS - community walkers [9]. The lesion level in MMC patients was reported intraoperatively and radiologically and scored from 1 to 3 ( 1 - thoracolumbar, 2 - lumbosacral, 3 - sacral lesion).

Image testing included kidney and urinary tract ultrasound. The following parameters were evaluated: length and width of the kidneys, bladder wall thickness and trabeculation, stasis at the renal pelvis and the calyces level, presence of hydronephrosis, parenchymal echogenicity, corticomedullary differentiation, and dilatation of perivesical fragments of the ureters. The parameters were evaluated separately for each kidney and subsequently added. Voiding cystourethrogram is the gold standard for evaluating and diagnosing vesicoureteral reflux. In our study, we analysed the presence of vesicoureteral reflux, its grade, laterality, and activity.

Urodynamics was performed in 114 cases, and its findings were classified as: neurogenic detrusor overactivity (NDO), areflexic bladder (AB), neurogenic detrusor-sphincter discoordination (NDSD), poor bladder wall compliance ( $\mathrm{PBC}$ ), and normal bladder function (NBF). Before cystometry, we also assessed the uroflowmetry parameters, including time to max flow rate (T max flow) and residual urine. Urodynamic study parameters, such as cystometric capacity, maximal detrusor pressure ( $\mathrm{p}$ det $\max$ ), detrusor pressure at urgency ( $\mathrm{p}$ det urg), and compliance, were analysed.

Statistical analysis was performed using the commercial package Statistica 13.0 (StatSoft Inc., Tulsa, OK, USA). Continuous variables were expressed as the median and range, unless stated otherwise. All studied parameters were analysed using nonparametric tests: Mann-Whitney, Kruskal-Wallis, and $\chi^{2}$ analysis. Multiple linear regression analyses were performed, including the GFR Schwartz and cystatin $\mathrm{C}$ as dependent variables, with gender, HS, presence of vesicoureteral reflux (VUR), and $\mathrm{p}$ det max considered as independent variables. Correlations were assessed with the Spearman test. Values of $p<0.05$ were considered significant. 
Patients and their caregivers were enrolled into the study after obtaining informed consent. The study protocol was approved by the Ethics Committee, Medical University of Bialystok, and was conducted in accordance with the Declaration of Helsinki.

\section{RESULTS}

The characteristics of the studied patients are presented in Table 1. The median age of the enrolled patients was nine years. There were statistically significant differences between girls and boys who were enrolled in the study, including increased echogenicity of the kidneys' parenchyma in the lower urinary tract ultrasound, cystometric capacity in urodynamics, and the presence of reflux in voiding cystourethrogram. We revealed that the highest prevalence of increased parenchyma in ultrasound was observed in boys with MMC, as well as larger cystometric capacity with a median of $171 \mathrm{ml}(20-700 \mathrm{ml})$, whereas girls' median cystometric capacity equalled $123 \mathrm{ml}$ (30-300 ml). Vesicoureteral reflux was observed in 30/99 (30\%) girls with MMC in contrast to $15 / 80$ (19\%) in boys with MMC.

Children with MMC were divided into three groups according to GFR Schwartz: I - glomerular hyperfiltration, II - normal GFR, and III - lower GFR. Patients from groups I and III were classified as those with renal deterioration. There were statistically significant differences between GFR Schwartz and lesion level as well as with ambulatory function of MMC patients defined by the HS $\left(\chi^{2}=6.64, p=0.04 ; \chi^{2}=8.99, p=0.01\right.$, respectively). More detailed results are presented in Table 2 . The lower the lev-

TABLE 1. Characteristics of 178 children with myelomeningocele

\begin{tabular}{|c|c|c|c|c|}
\hline Characteristics & MMC Patients & MMC Girls & MMC Boys & Comparison \\
\hline \multicolumn{5}{|c|}{$n(\%)$} \\
\hline Female/Male & 178 & $99(56)$ & $79(44)$ & \\
\hline \multicolumn{5}{|c|}{ median (min-max) } \\
\hline Age (years) & $9(1-18)$ & $7.2(1-18)$ & $9.67(1-18)$ & 0.13 \\
\hline Height (cm) & $121(52-177)$ & $29(70-177)$ & $130(52-174)$ & 0.13 \\
\hline Weight (kg) & $25.2(4.7-84.8)$ & $21(5.5-70)$ & $29.7(4.7-84.8)$ & 0.23 \\
\hline BMI $\left(\mathrm{kg} / \mathrm{m}^{2}\right)$ & $17.9(9.6-37.2)$ & $16.9(10.71-37.2)$ & $19.16(9.6-31.5)$ & 0.3 \\
\hline \multicolumn{5}{|l|}{ Hoffer scale (HS) } \\
\hline $1 \mathrm{HS}$ & $99(62)$ & $49(58)$ & $50(68)$ & 0.17 \\
\hline $2 \mathrm{HS}$ & $22(14)$ & $12(14)$ & $10(13.5)$ & 0.89 \\
\hline $3 \mathrm{HS}$ & $18(11)$ & $14(16)$ & $4(5)$ & $0.03^{*}$ \\
\hline $4 \mathrm{HS}$ & $20(13)$ & $10(12)$ & $10(13.5)$ & 0.77 \\
\hline \multicolumn{5}{|l|}{ Lesion level } \\
\hline Thoracolumbar & $44(29)$ & $21(24)$ & $23(31)$ & 0.53 \\
\hline Lumbosacral & $92(61)$ & $46(52)$ & $46(62)$ & 0.54 \\
\hline Sacral & $16(10)$ & $11(13)$ & $5(7)$ & 0.05 \\
\hline Number of CICs performed per day & $4(0-8)$ & $4(0-8)$ & $4(0-7)$ & 0.07 \\
\hline Cystatin C (mg/l) & $0.32(0.092-4.98)$ & $0.32(0.092-4.98)$ & $0.33(0.16-1.5)$ & 0.87 \\
\hline Serum creatinine (mg/dl) & $0.35(0.058-8.57)$ & $0.33(0.058-8.57)$ & $0.36(0.17-5.25)$ & 0.78 \\
\hline Serum urea $(\mathrm{mg} / \mathrm{dl})$ & $26(7.2-212)$ & $28(7.2-212)$ & $25(8-57.7)$ & 0.25 \\
\hline GFR Schwartz formula (ml/min/1.73m²) & $142(4.58-301)$ & $146.55(4.58-264.32)$ & $139.08(37.54-301)$ & 0.74 \\
\hline
\end{tabular}

TABLE 2. Glomerular filtration rate Schwartz assorted by Hoffer scale groups

\begin{tabular}{|l|c|c|c|c|}
\hline \multirow{2}{*}{ Median (min-max) } & \multicolumn{4}{|c|}{ Hoffer scale } \\
\cline { 2 - 5 } & 1 1HS & 2HS & 3HS & 4HS \\
\hline GFR Schwartz $\left(\mathrm{ml} / \mathrm{min} / 1.73 \mathrm{~m}^{2}\right)$ & 157.98 & 147.24 & 142 & 130.3 \\
& $(4.58-301.5)$ & $(58.1-208.3)$ & $(35.7-224.5)$ & $(68.5-214.8)$ \\
\hline
\end{tabular}

GFR - glomerular filtration rate, $\mathrm{HS}$ - Hoffer scale 
TABLE 3. Urodynamic findings according to Hoffer scale groups

\begin{tabular}{|l|c|c|c|c|c|}
\hline \multirow{2}{*}{ Urodynamic findings } & \multirow{2}{*}{$n(\%)$} & \multicolumn{4}{|c|}{ Hoffer scale $n(\%)$} \\
\cline { 3 - 6 } & & 1 1HS & 2 HS & 3 HS & 4 HS \\
\hline ND0 & $67(59)$ & $43(64)$ & $12(18)$ & $5(7)$ & $7(11)$ \\
\hline NDSD & $12(10)$ & $6(50)$ & $1(8)$ & $3(25)$ & $2(17)$ \\
\hline AB & $19(17)$ & $11(58)$ & $3(16)$ & $3(16)$ & $2(10)$ \\
\hline PBC & $12(10)$ & $7(58)$ & 0 & $3(25)$ & $2(17)$ \\
\hline NBF & $4(4)$ & $2(50)$ & $1(25)$ & 0 & $1(25)$ \\
\hline
\end{tabular}

HS - Hoffer scale, NDO - neurogenic detrusor overactivity, NDSD - neurogenic detrusor-sphincter discoordination, AB - areflexic bladder, PBC - poor bladder wall compliance, NBF - normal bladder function

TABLE 4. Linear regression analysis for glomerular filtration rate Schwartz and cystatin C

\begin{tabular}{|l|c|c|c|c|}
\hline \multirow{2}{*}{ Clinical parameters } & \multicolumn{2}{|c|}{ GFR Schwartz } & \multicolumn{2}{c|}{ Cystatin C } \\
\cline { 2 - 5 } & Standardised $\beta$ & $p$-value & Standardised $\beta$ & $p$-value \\
\hline Gender (male vs. female) & -5.064 & 0.694 & 0.035 & 0.45 \\
\hline Hoffer scale (1 vs. 4) & 34.908 & 0.216 & 0.081 & 0.375 \\
\hline Hoffer scale (2 vs. 4) & 21.684 & 0.47 & 0.152 & 0.125 \\
\hline Hoffer scale (3 vs. 4) & 22.188 & 0.378 & 0.162 & 0.051 \\
\hline Presence of VUR (with vs. without) & -37.593 & 0.021 & 0.010 & 0.86 \\
\hline$p$ det max (continuous) & -0.079 & 0.660 & 0.002 & $<0.001$ \\
\hline
\end{tabular}

GFR - glomerular filtration rate, VUR - vesicoureteral reflux, p det max - maximal detrusor pressure

el of the lesion and the worse the ambulatory function, the higher the GFR Schwartz (hyperfiltration). Ultrasonography parameters, including stasis at the renal pelvis and the calyces level, the presence of hydronephrosis, loss of the corticomedullary differentiation, increased parenchymal echogenicity and trabeculation of the bladder wall, varied significantly between the GFR groups $\left(\chi^{2}=7.19\right.$, $p=0.03 ; \chi^{2}=15.47, p=0.0004 ; \chi^{2}=11.99, p=0.0025$; $\chi^{2}=36.75, p<0.001 ; \chi^{2}=9.37, p=0.0092$, respectively). If the above-mentioned parameters were present in renal ultrasonography, lower GFR Schwartz was observed. High $\mathrm{T}$ max flow and the presence of postvoid residual urine in uroflowmetry also correlated with lower GFR Schwartz. Negative correlations were found between serum concentrations of cystatin C, creatinine, urea, and GFR Schwartz $(r=-0.43, r=-0.76, r=-0.39$, respectively; $p<0.05)$.

\section{ULTRASONOGRAPHY}

There were no statistically significant differences in the ultrasonography parameters between the HS groups, excluding the corticomedullary differentiation $\left(\chi^{2}=11.09\right.$, $p=0.01$ ). The worse the ambulatory function, the more often the corticomedullary differentiation was obliterated. We revealed that bladder trabeculation coexisted more often with stasis at the renal pelvis and the calyces $(r=0.3, p<0.05)$, the presence of hydronephrosis $(r=0.455, p<0.05)$, and increased parenchymal echogenicity $(r=0.295, p<0.05)$. We observed that children with bladder trabeculation, detected in ultrasound, were significantly older compared than those without trabeculation.

\section{VOIDING CYSTOURETHROGRAM}

A total of 35/146 (24\%) patients had VUR, and 10/146 (6.8\%) of them had refluxes diagnosed in the past that resolved and were low-grade (I, II, III grade). Left sided reflux was noted more frequently - 33/45 (73\%). We observed that loss of the corticomedullary differentiation and increased parenchymal echogenicity in ultrasound were more frequently seen in children with VUR $(r=-0.243$, $r=-0.216$, respectively; $p<0.05$ ).

\section{URODYNAMICS}

The characteristics of the urodynamic findings according to the HS groups are shown in Table 3. No statistically significant differences were found according to the HS. In all four groups, the most common diagnosis was NDO. However, the HS correlated with urodynamic parameters: positively with uroflowmetry $\mathrm{T}$ max flow $(r=0.49, p<0.05)$ and voided volume $(r=0.49, p<0.05)$, and negatively with $\mathrm{p}$ det $\max (r=-0.21, p<0.05)$. P det max statistically significantly correlated with kidney and urinary tract ultrasound parameters: trabeculation, stasis at the renal pelvis and the calyces level, the presence of hydronephrosis, corticomedullary differentiation, and parenchymal echogenicity $(r=-0.23, r=-0.22, r=-0.3$, $r=-0.23, r=-0.25$, respectively; $p<0.05)$. The higher the $\mathrm{p}$ det max, the more changes in renal imaging in ultrasound of the kidney and urinary tract were found.

We assessed the number of nephrological follow-up visits and the number of performed urodynamics for 
every child with MMC during the study period. A negative correlation was found between GFR Schwartz and the number of nephrological follow-up visits $(r=-0.17$; $p<0.05)$, in contrast to the number of performed urodynamics, which positively correlated with GFR Schwartz $(r=0.3 ; p<0.05)$. We also recorded the fact that children who had urodynamic assessment performed more frequently, less often had ultrasonography abnormalities, such as: stasis at the renal pelvis and the calyces, the presence of hydronephrosis, and increased parenchymal echogenicity. Negative correlations were found between serum concentrations of cystatin $\mathrm{C}$ and creatinine, and the number of performed urodynamic studies $(r=-0.62$, $r=-0.24$, respectively; $p<0.05)$.

Because of the effects of gender, HS, presence of VUR, and urodynamic parameters, especially p det max on renal deterioration, detected in our study, we performed two models of multivariable linear regression. In the first model GFR Schwartz was the dependent variable, and in the second - cystatin C. We selected statistically significant explanatory variables such that the sum of squared residuals would be minimal in the model.

Multiple linear regression analysis with the GFR as the dependent variable was not statistically significant $(F=1.19, p=0.31)$ and accounted for $12 \%$ of the variations of GFR Schwartz ( $r 2=0.123$ for the model). Only one significant relationship for the GFR Schwartz was noted with the presence of VUR $(\beta=-0.27, t=-2.35, p=0.02)$. The second model with the remaining four parameters as independent values was statistically significant $(F=3.33$, $p<0.001)$ and accounted for more than $41 \%$ of the variations of cystatin $\mathrm{C}(r 2=0.417$ for the model). A strong relationship between $\mathrm{p}$ det max and cystatin $C$ level was recorded $(\beta=-0.002, t=4.02, p<0.001)$. The results of the regression are summarised in Table 4 . It showed that the presence of high-grade VUR and high p det max are the most important predictors of renal deterioration in MMC children.

\section{DISCUSSION}

Myelomeningocele is the leading cause of congenital neurogenic bladder worldwide, which is an important risk factor of secondary upper urinary tract damage. At birth, most newborns with MMC have a normal upper urinary tract [9-11]. However, according to studies the majority of them will develop deterioration of renal function without adequate management. Both of the paediatric nephrology departments in which our study was performed are responsible for comprehensive renal function follow-up of children with MMC, starting early in the course of the disease.

The available studies have aimed at establishing an association between renal injury and typically performed nephrourological assessments. Arora et al. [10] cited a variety of potential risk factors of renal injury for MMC, in- cluding increasing age, evidence of hydroureteronephrosis and vesicoureteral reflux, high leak pressures, low bladder volume, and serum creatinine $>1 \mathrm{mg} / \mathrm{dl}$ at presentation. In our study, we also observed a relation between increasing age and the renal image in ultrasound. Children with bladder trabeculation detected in ultrasound were significantly older compared to those without trabeculation.

The presence of VUR seems to be an important risk factor of renal deterioration. In our study, the higher the VUR grade, the lower the GFR Schwartz score. However, voiding cystourethrogram and ultrasonography can only identify upper tract abnormalities once they have already occurred. To ascertain the safety of the upper urinary tract it is crucial to control low bladder pressures in a urodynamic study [11-14]. In our study, we found that the higher the $\mathrm{p}$ det max, the more changes in the renal imaging in the ultrasound of the lower urinary tract were found. High T max flow in uroflowmetry and the presence of postvoid residual urine volume in urodynamics also correlated with lower GFR Schwartz. We recommend an initial urodynamic study at the early stages of the disease as well as follow-up urodynamics to monitor response to therapy and to identify the high-risk group of patients. Pharmacological treatment of increased bladder pressures may be the key to preventing renal deterioration in MMC patients. In our study children who had urodynamic assessment performed more frequently, less often had ultrasonography abnormalities, had lower creatinine and cystatin C levels, and greater GFR Schwartz. With regard to these results, we recommend frequent urodynamics as an important tool to estimate urinary tract function in children with MMC.

Due to our study hyperfiltration, defined as GFR Schwartz $>140 \mathrm{ml} / 1.73 \mathrm{~m}^{2} / \mathrm{min}$, seems to be a risk factor for renal deterioration in children with MMC. It correlated with the HS. We state that wheelchair-dependent patients are at risk for unsafe storage bladder pressures, which can be found and monitored on urodynamics.

Our list of potential risk factors of renal deterioration in children with MMC: abnormalities in ultrasound, the presence of high-grade VUR in cystogram, increased bladder pressures on urodynamics, hyperfiltration, and increased levels of cystatin C. We recommend frequent urodynamic assessment in children with MMC to prevent renal deterioration.

This study has some limitations which have to be pointed out. The imprecise calculation of GFR using the Schwartz formula due to muscle mass deficits in patients with MMC and possible differences in laboratory methods and imaging techniques between both centres are the major limitations. Larger series are needed to confirm our results.

\section{CONCLUSIONS}

Potential risk factors of renal deterioration in children with MMC are abnormalities in ultrasound, the presence 
of high grade VUR in cystogram, increased bladder pressures on urodynamics, hyperfiltration, and increased levels of cystatin C.

Urodynamic evaluation may play a role in the management of children with MMC by identifying those with increased bladder pressures.

Frequent urodynamic assessment may contribute to prevention of renal function in children with MMC.

\section{DISCLOSURE}

The authors declare no conflict of interest.

\section{REFERENCES}

1. Luz CL, Moura MCDS, Becker KK, et al. The relationship between motor function, cognition, independence and quality of life in myelomeningocele patients. Arq Neuropsiquiatr 2017; 75: 509-514.

2. Korzeniecka-Kozerska A, Kulesza-Bronczyk B, Okurowska-Zawada B, et al. Clinical characteristics of patients with neurogenic bladder due to myelomeningocele - Eighteen-year retrospective study; University Children's Hospital, Bialystok, Poland. Pediatr Pol 2015; 90: 283-289.

3. Maison POM, Lazarus J. The management of paediatric neurogenic bladder: an approach in a resource-poor setting. Paediatr Int Child Health 2017; 17: 1-6.

4. Korzeniecka-Kozerska A, Porowski T, Bagińska J, et al. Urodynamic Findings and Renal Function in Children with Neurogenic Bladder after Myelomeningocele. Urol Int 2015; 95: 146-152.

5. Sinha S. Follow-up urodynamics in patients with neurogenic bladder. Indian J Urol 2017; 33: 267-275.

6. Danforth TL, Ginsberg DA. Neurogenic lower urinary tract dysfunction: how, when, and with which patients do we use urodynamics? Urol Clin North Am 2014; 41: 445-452.

7. Miklaszewska M, Korohoda P, Zachwieja K, et al. Can We Further Improve the Quality of Nephro-Urological Care in Children with Myelomeningocele? Int J Environ Res Public Health 2016; 13: 876.

8. Korzeniecka-Kozerska A, Liszewska A. Inflammatory and oxidative status in neurogenic bladder children after meningomyelocele. Prog Health Sci 2015; 5: 22.

9. Hoffer MM, Feiwell E, Perry R, et al. Functional ambulation in patients with myelomeningocele. J Bone Join Surg Am 1973; 55: 137-148.

10. Arora G, Narasimhan KL, Saxena AK, et al. Risk factors for renal injury in patients with meningomyelocele. Indian Pediatr 2007; 44: 417-420.

11. Ozel SK, Dokumcu Z, Akyildiz C, et al. Factors affecting renal scar development in children with spina bifida. Urol Int 2007; 79: 133-136.

12. Filler G, Gharib M, Casier S, et al. Prevention of chronic kidney disease in spina bifida. Int Urol Nephrol 2012; 44: 817-827.

13. Olandoski KP, Koch V, Trigo-Rocha FE. Renal function in children with congenital neurogenic bladder. Clinics 2011; 66: 189-195.

14. Filler G, Gharib M, Casier S, et al. Prevention of chronic kidney disease in spina bifida. Int Urol Nephrol 2012; 44: 817-827. 